\title{
Lab VIEW Programming for Vibration Analysis
}

\author{
A.K.Desai, A.G.Bharate,V.P.Rane, S.V.Gholap,A. C. Mitra \\ (Department of Mechanical Engineering, M.E.S. College of Engineering, Pune, S.P. Pune University, India)
}

\begin{abstract}
Lab VIEW (Laboratory Virtual Instrument Engineering Workbench) is a graphical programming language mainly used for data Acquisition, measurement and analysis. In this study the designing of a system using Lab VIEW for the measurement and analysis of vibration was done. The software part consists of programming of Data Acquisition and signal processing of signals for vibration measurement.In this programming the DAQ(Data Acquisition) input is in Time domain. Using FFT(Fast Fourier Transform) analyzer program convert the time domain graph into the frequency domain and finally it pick-up the frequency corresponding to the highest amplitude or peak amplitude using an peak detector after FFT analyzer. Experimental results shows that the study and analysis of vibration of any machines using Lab VIEW programming is more simple, good and visual than any other text language and this vibration testing system not only can perform real time data acquisition of multiple channels signal but also has good expansibility, high speed and friendly interface.
\end{abstract}

Keywords-Data Acquisition System, LabVIEW, Vibration signal, FFT, Graphical programming, Virtual instrument, Peak detector, Signal analysis.

\section{INTRODUCTION}

Now a days there is a need to measure vibration as the machine life is extended because of elimination of vibration. The repairing and maintenance of machine depends on time period not on condition of the machine [1]. Vibration can also produce a noise which is undesirable hence it also helped to reduce the noise. Analysis and Monitoring of signal are required for the fault detection analysis of any machine, engine as well as its graphical representation are used for the measurement of earthquake amplitude, etc. Vibration measurement can be used in many applications like industrial machine, domestic machine, vehicle engine in aerospace and earthquake measurement, etc. Vibration measurement can also be used to analyze engine performance [2].

The information about a fault feature of the equipment are built-in vibration signal. Therefore the measurement and analysis of vibration are necessary. The type of vibration measuring instrument depends on range of frequency and amplitude, size of the machine, condition of operation of the machine.The vibration is measured by the various types of transducer like variable resistance transducer, piezoelectric transducer, electrodynamic transducer. These transducers further connected to the DAQ. Piezoelectric transducer provides a accurate result than the other transducer. The accelerometer is the piezoelectric transducer which provides acceleration data of an object to the DAQ and further it is provided with the software. Acceleration can be motion, shock or vibration. Different type of accelerometer is used depending on the frequency range and acceleration type. In general piezoelectric accelerometer is used to measure the vibration [2].

The software uses to measure the vibration are DTS Slice Data acquisition system, Vibrometra, OROS analyzers, Vibration VIEW software, UEI Spectrum Analyzer. NI Lab-VIEW is the software which is based on graphical language. These VIs permit us to utilize established advanced digital signal processing without composing a single line of code.In this paper with the help of Lab-VIEW blog programming was developed for the mechanical vibration test analysis. The output panel of software shows the time domain graph. The time domain graph cannot gives mainly detail which can be obtained from frequency domain so that by using an FFT analyzer time domain is converted into the frequency domain.. The system can receive the vibration from accelerometer which is further given to the DAQ in signal form. DAQ is connected to the NI Lab-View software and with the help of peak detector frequency domain graph shows the maximum amplitude with corresponding frequency.

\section{HARDWARE SETUP}

The fundamental block diagram of the setup is shown in Fig.1 It contains a vibration sensor / transducer, data acquisition device and computer. The transducer collects the signals and converts it from nonelectric to electric form. The piezoelectric accelerometers connected to four different channels of 9234 DAQ module. In the present study two different piezoelectric accelerometers were connected to two channels of module. One was uni-axial 351B03 accelerometer and another was tri-axial 354C03 accelerometer. The function 
of the module is coupling the data of all active channels and conditioning them. The module was coupled with chassis and its function is synchronizing and transferring data from the input of module to an external host. The data were analyzed in LabVIEW programming in a computer.

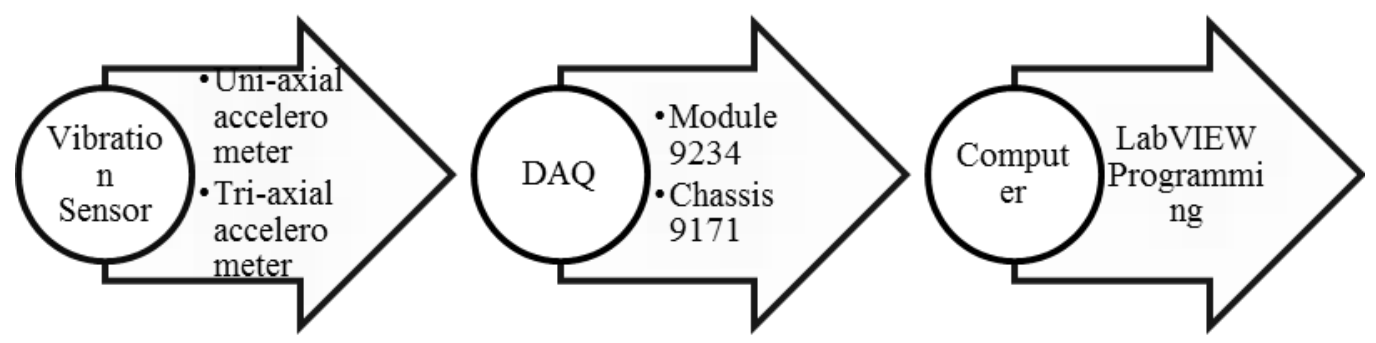

Fig.1: Block diagram

\section{LABVIEW PROGRAMMING}

In the present study, the LabVIEW programming was developed for vibration analysis in which the detecting of maximum amplitude and respective frequency as well as picking all peaks above the threshold value. By using this program the frequency and amplitude were represented on graph due to that time and frequency domain easily visualized and understood. The programming shown in Fig 2. And components were explained in below.

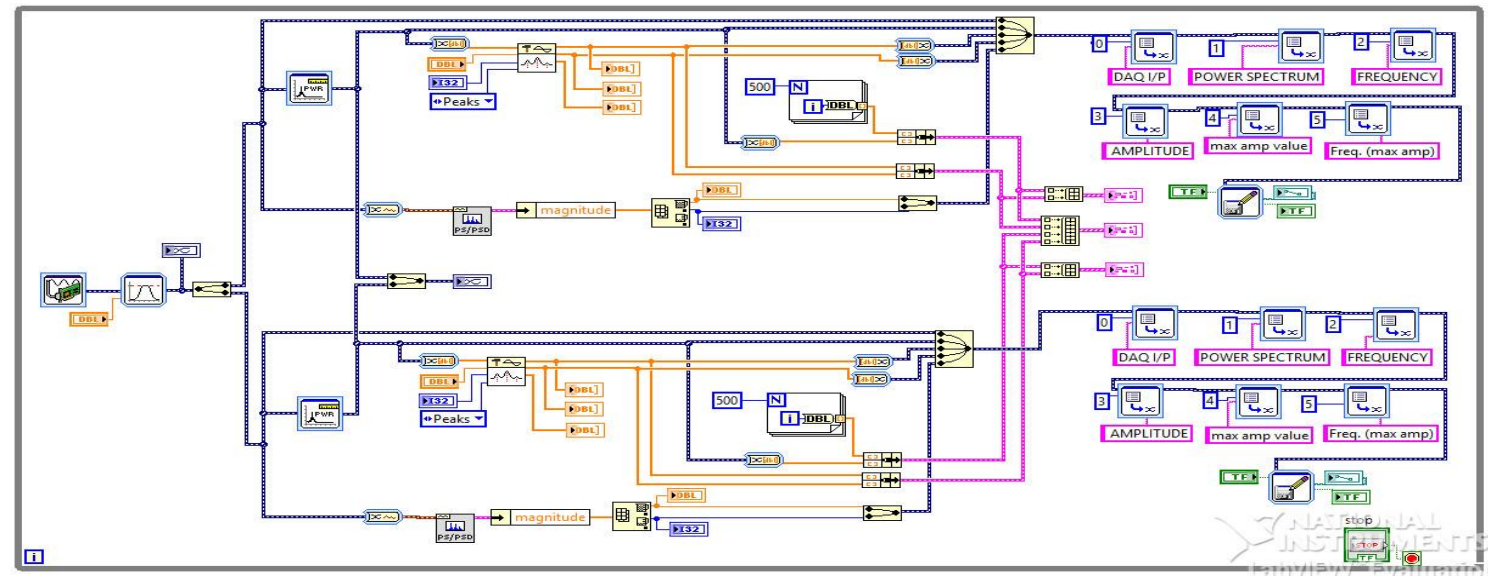

Fig.2: Labview Programming

\subsection{DAQ Assistant And Signal Filtering}

The signals coming from DAQ were collected by a DAQ assistant in programming. In the DAQ assistant the different type of input signals were given and signals from the various channels are segregated. The dynamic data carried by wires from DAQ assistant to filter. In programming highpass infinite impulse response filter with Butterworth topology were used. The filter used to eliminate the low frequency signals coming from the transducer. The output filtered data displayed by mixed signal graph which is connected to the output of filter as shown in fig3.

\subsection{Spectral Measurement And Mixed Signal Graph}

The filtered data were coming for spectral measurement in FFT analyzer. In the FFT the incoming time domain data were converted into the frequency domain. Any wave or signal data were a series combination of amplitude, frequency and time. Spectral analysis takes the individual sine wave for further analysis. And the outcome results come in the frequency domain in which amplitude is a function of frequency. In this study, two FFT analyzers were used

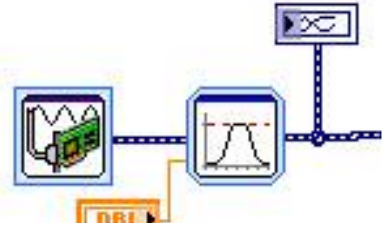

Fig 3DAQ assistant and signal filtering

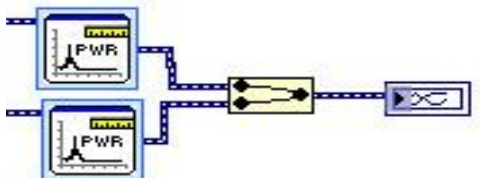

Fig 4 spectral measurement and mixed signal graph for analyzing two different analog data shown in fig4. The output of the FFT was observed in graphical presentation using a mixed signal graph. 


\subsection{Peak Detector}

Peak detector was used to detect all peaks present in the output of the FFT. The data type converter is used for converting dynamic data to array type data because the input to peak detector needs array type data. The parameter for peak detectors was given by the operator as per the need of an operator. The threshold was one of the parameters by which a lower limit will set and above that limit all peaks are detected by the peak detector shown in fig5. The output of peak detector was peak amplitude values, the location where peak amplitudes were detected and $2^{\text {nd }}$ derivative of amplitude.

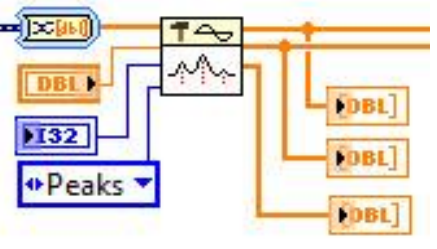

Fig.5 peak detector

\subsection{Power Spectrum And Maximum Value Detection}

The maximum amplitude was detected among given input data. For that the programming shown in fig6 was used to detect the maximum value of amplitude. For that power spectrum (PS) is used to convert data into the frequency domain for easy detection of amplitude to function of frequency. The data from PS to unbundle by names carried by cluster carrying data type wire. The unbundle by names was used for segregating data into elements. The array max \& min was used for required result.

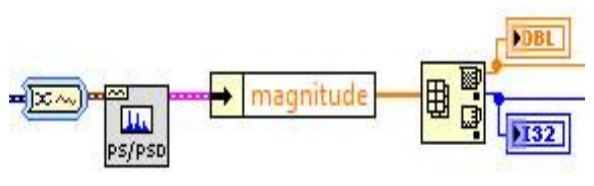

Fig 6 power spectrum and maximum value detection

\subsection{Multiple Graph Plot}

For visualization of results the graphical presentation is important. For that, the multiple graph plot was used. For this the cluster type data was required. By using dynamic data convertor data is converted from dynamic to array type data and using bundles array type data is converted into cluster type data. This process was shown in fig. The for loop was used for a plotting number of results in graph same as the length given to the loop. The build array was used for combining all cluster data and give appended array for plotting a graph.

\subsection{Write To Measurement File}

For saving data and results in LabVIEW, there is the programming which is shown in fig. The merge signal is used for merging all signals for saving back to back within same format and the same file. The set dynamic data attributes are used to collect single data coming from merging signal and send the remaining data to next attribute. In the set dynamic data attribute, the signal index was an important factor as per that control the data was taken by the attribute and save it. The write to measurement used to save files in the format of .xlsx and LVM.

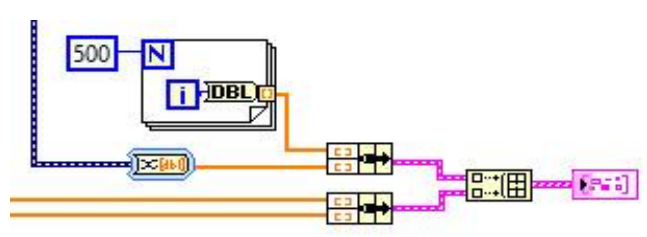

Fig.7 multiple graph plot

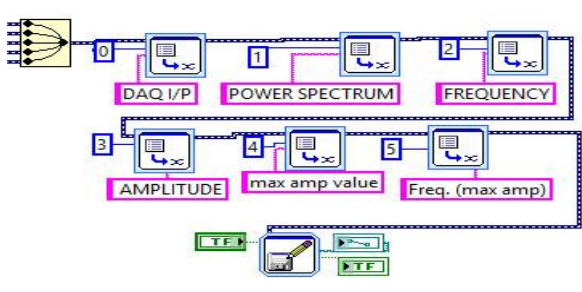

Fig. 8 write to measurement file

\section{EXPERIMENTAL SETUP}

The primary objective of this thesis was to study the feasibility of Lab-VIEW using an accelerometer to monitor vibration signatures of a machine. In order to test the Lab-VIEW programming, mechanical vibration testing system is arranged on CHOP-SAW machine as shown in Fig9.In the test experimentation, two accelerometers are used as a vibration transducer. One of them is attached directly on handle of CHOPSAW machine and another one is attached on the handle of CHOPSAW machine having vibration isolation coating. These accelerometers are then connected to the DAQ and it to the computer having Lab-VIEW programming. Vibration results were taken while cutting the MS rod on CHOP-SAW machine. The results were saved in excel format using a write to measurement file. By comparing results from frequency domain, it can be easily analyzed the effect of vibration on machines. Finally, it shows that, using the Lab-VIEW programming it can measure the real time vibration signals generated by CHOPSAW machine which will be useful in making key decision for maintenance of the machine. 


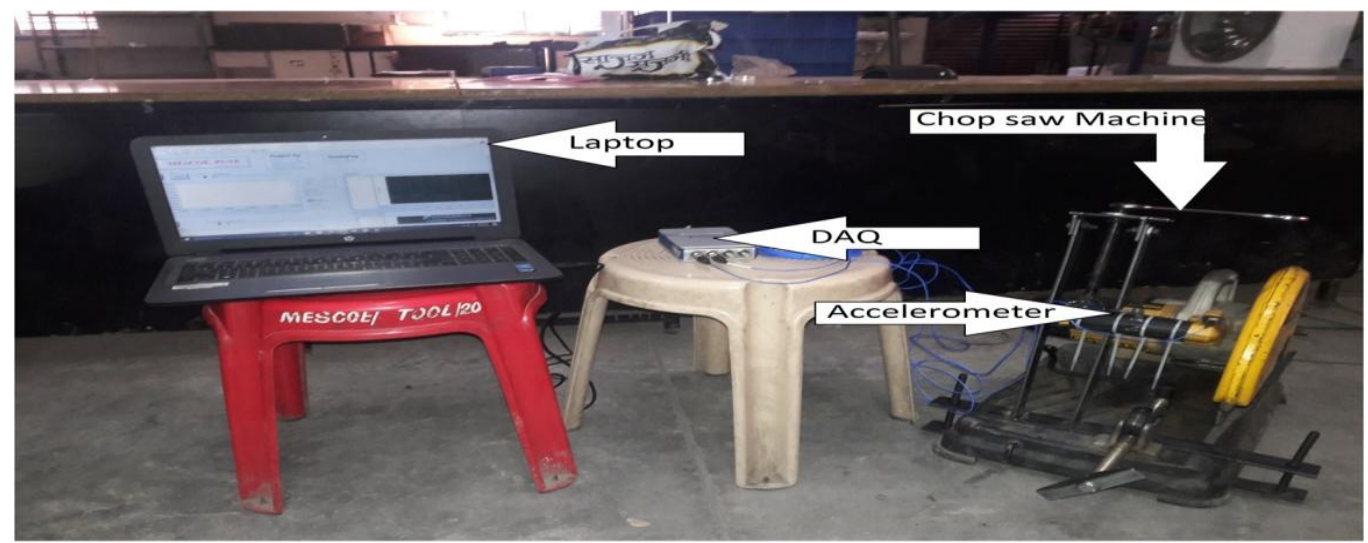

Fig.9 Experimental set-up for testing of the system

\section{RESULTS AND DISCUSSION}

To measure the vibration and to plot the time domain and frequency domain, the vibration transducer that is accelerometer is fixed on on the industrial machine which converts the acceleration into an analog signals. Vibration signal acquired from the CHOPSAW machine was given to the Lab-VIEW Programming and Lab VIEW front panel is as shown in Fig10. Fig.11. and Fig12.

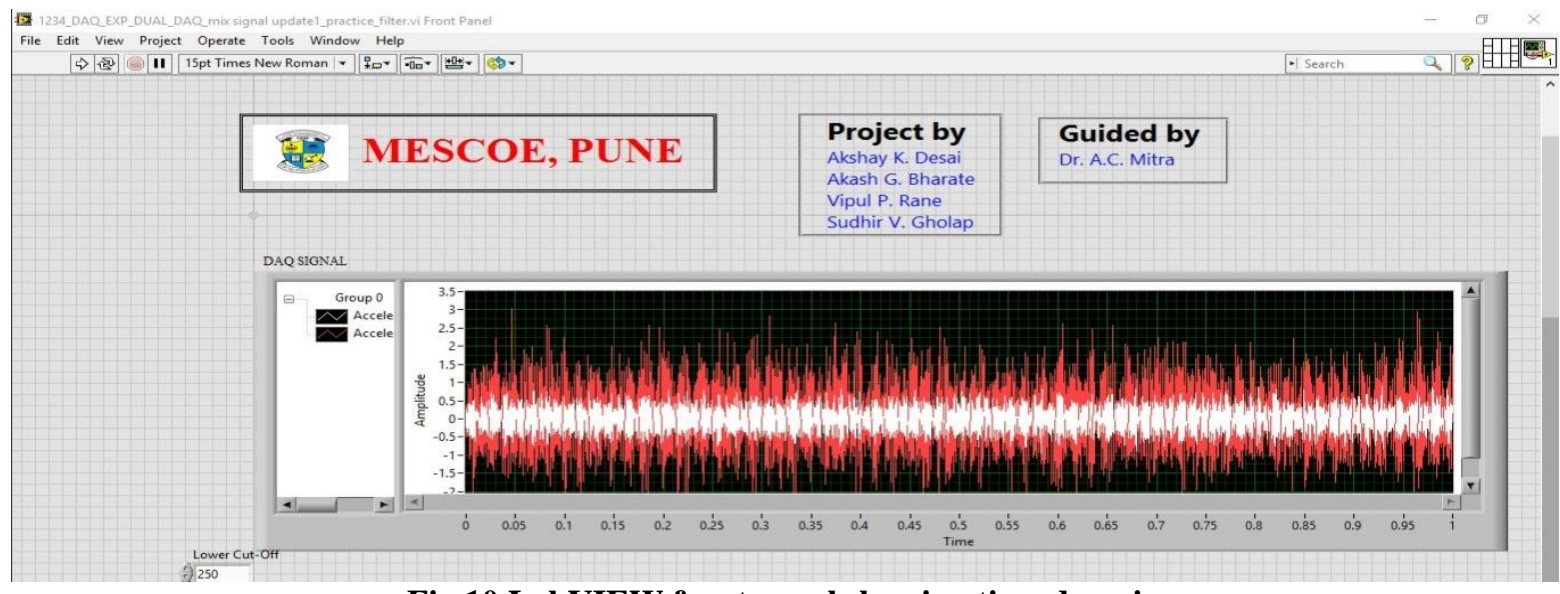

Fig.10 LabVIEW front panel showing time domain

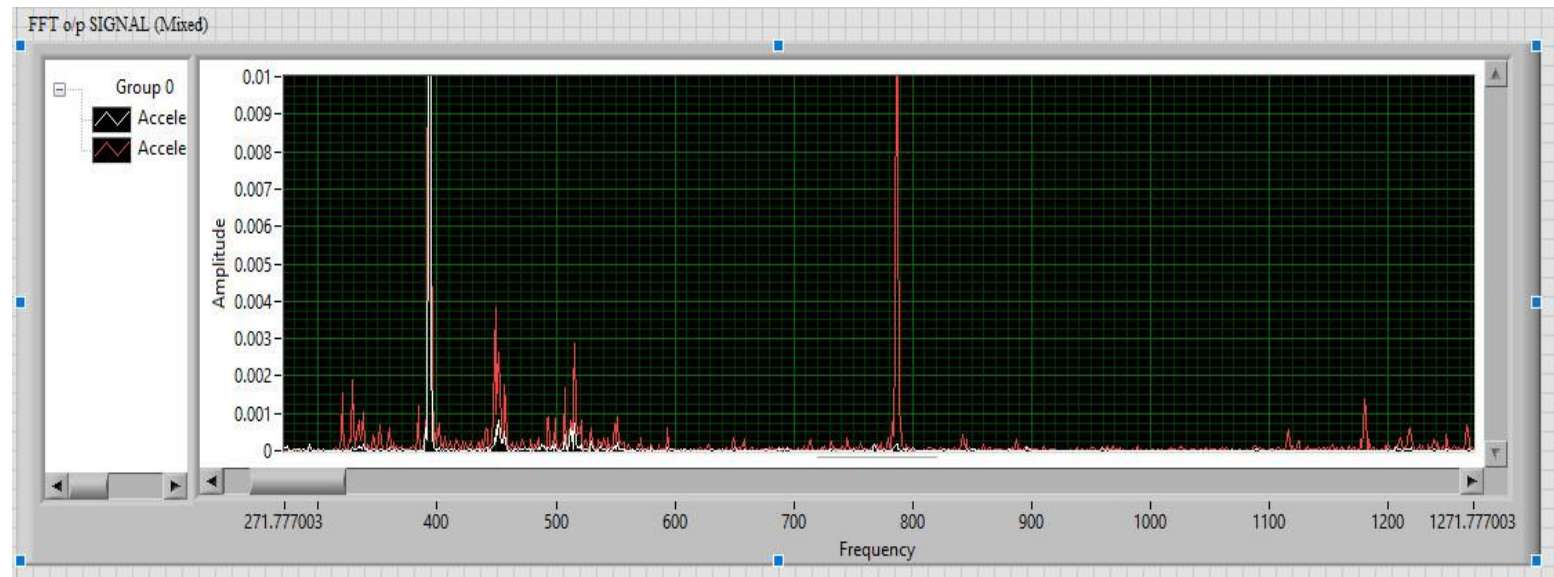

Fig.11 LabVIEW front panel showing frequency domain 


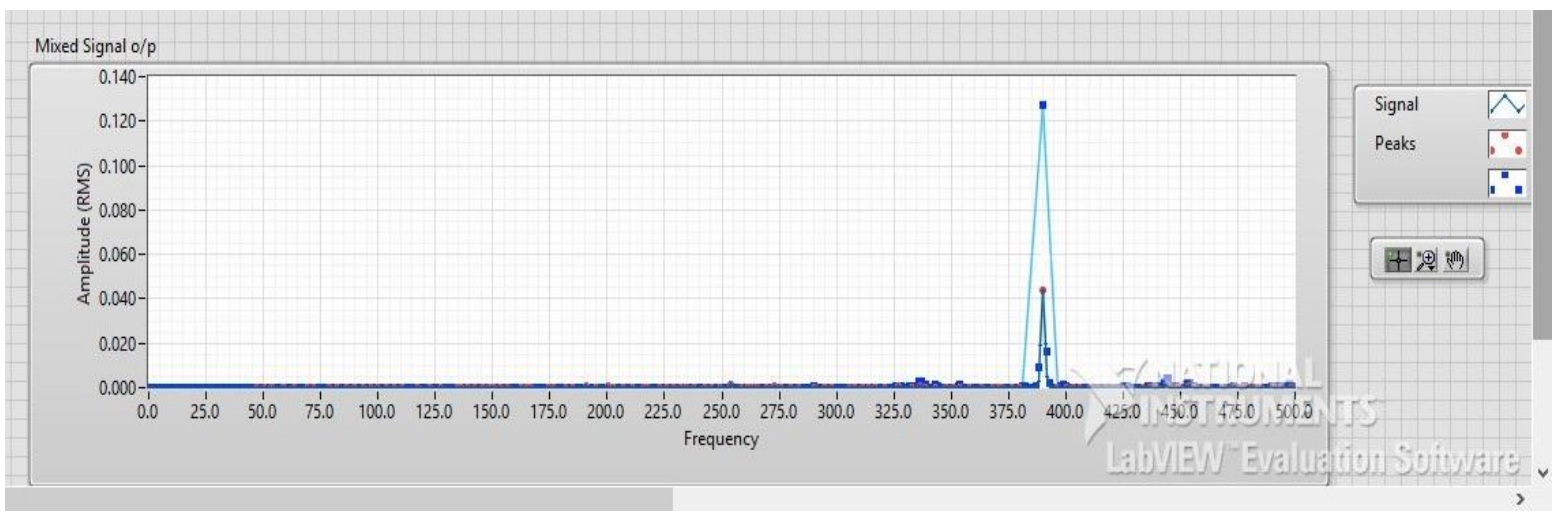

Fig.12 LabVIEW front panel showing frequency domain with peak detection

Fig 10 shows the DAQ input in Time domain. And for frequency analysis, program converts the time domain graph into the frequency domain using an FFT analyzer which was shown in Fig 11. and finally it pickup the frequency corresponding to the highest amplitude or peak amplitude using a peak detector after FFT analyzer which was shown in Fig. 12. Comparing the results which were getting from Lab-VIEW programming it concludes that the vibration amplitude on the handle is more than the vibration amplitude on the handle covered with vibration isolator. By comparing results from frequency domain, it can be easily analyzed the effect of vibration on machines.

\section{CONCLUSION}

In present study, blog programming was developed in NI Lab VIEW software to design a vibration testing platform for sampling of vibration signal and data processing. By experimenting with the constructed hardware system, the results indicate that Lab VIEW programming gives an valid result and it is more simple, visual and reliable than other text languages. This vibration testing system has expansibility, rapid, high precision and friendly interface. The analysis of vibration and monitoring the system for mechanical vibration presented has the main advantage his flexibility. The outcomes demonstrate that Lab VIEW is more straightforward, visual and simple than other programming language.Because of the graphical programming environment, LabVIEW is suitable for modern computer-based instrumentation systems, whether for data acquisition and control or test and measurement.

\section{REFERENCES}

[1] H.S.Shah,P.N.Patel,S.P.Shah,M.T.Thakker, 8 channel vibration monitoring and analyzing system using LabVIEW, Engineering (NUiCONE), Nirma University International Conference on. IEEE, 2013, 1-4.

[2] I.A.Jamil,M.I.Abedin,D.K.Sarker,Vibration data acquisition and visualization system using MEMS accelerometer, International Conference on Electrical Engineering and Information \& Communication Technology (ICEEICT), IEEE, 2014.

[3] L.Lita,D.A.Visan,G.Mujea,G.Ghita, LabVIEW application for analysis of mechanical vibrations from industrial environment,Electronics Technology: Meeting the Challenges of Electronics Technology Progress,28th International Spring Seminar on. IEEE, 2005

[4] A.Gani, Asan,M. J. E. Salami, A LabVIEW based data acquisition system for vibration monitoring and analysis ,Research and Development, SCOReD 2002. Student Conference on. IEEE, 2002.

[5] Z.Hua, Application of LabVIEW in the design of data acquisition and signal processing system of mechanical vibration , Mechatronic Science, Electric Engineering and Computer (MEC), 2011 International Conference on. IEEE, 2011.

[6] Kelly Graham S, Fundamentals of Mechanical Vibrations(McGraw Hill Inc., New York.2002)

[7] www.ni.com accessed on 10/10/2016 